$$
\text { "tmcs-szilasi" — 2012/3/1 — 0:14 - page } 175 \text { — \#1 }
$$

\title{
Classical theorems on hyperbolic triangles from a projective point of view
}

\author{
Zoltán Szilasi
}

Abstract. Using the Cayley-Klein model of hyperbolic geometry and the tools of projective geometry, we present elementary proofs for the hyperbolic versions of some classical theorems on triangles. We show, in particular, that hyperbolic triangles have no Euler line.

Key words and phrases: Cayley-Klein plane, hyperbolic triangles, Euler line.

ZDM Subject Classification: G90.

\section{Introduction}

In the training of the prospective mathematics teachers hyperbolic geometry plays (or should play) an important role. Historically, it is a counterexample for Euclidean geometry, and has an equally rich structure. This richness makes hyperbolic geometry also an active area of contemporary mathematics. Pedagogically, it suggests very efficiently that mathematical thinking relies only on axioms and deduction rules, and it is independent of what we see - sometimes it contradicts our view.

It was a great challenge also for the greatest mathematicians to find out and prove that the axiom of parallels is not necesserily true in the Euclidean way, and a meaningful geometry can be built from its negation as well. For the students, the Cayley-Klein model very convincingly demonstrates an absolute geometry where the Euclidean axiom of parallels does not hold.

Copyright (C) 2012 by University of Debrecen
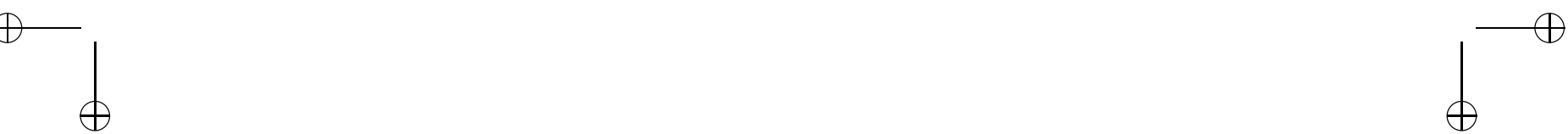


$$
\text { "tmcs-szilasi" — 2012/3/1 — 0:14 — page } 176 \text { — \#2 }
$$

In this paper we present an elementary exposition of some classical theorems of hyperbolic geometry assuming a solid knowledge of projective geometry. It turns out that in hyperbolic geometry a useful method of solving problems is to translate them into the language of projective geometry. Our theorems also give an opportunity for comparing Euclidean and hyperbolic geometry, since most of them are true in the Euclidean plane as well, but our last theorem concerning the Euler line indicates an interesting difference between Euclidean and hyperbolic triangles.

\section{Preliminaries}

Let a nondegenerate conic be given in the real projective plane. We call this conic the absolute conic. The inner points of the conic are called hyperbolic points, and the subsets of the projective lines consisting of hyperbolic points are the hyperbolic lines. The original projective line is called the projective line corresponding to the hyperbolic line. Let the isometries be the restrictions of the automorphisms of the given conic to the set of hyperbolic points. Thus we obtain an absolute plane, called the Cayley-Klein plane. It can be shown that every hyperbolic plane is isomorphic to the Cayley-Klein plane.

We say that a set of hyperbolic lines forms a pencil if the corresponding projective lines are concurrent. If their common point is a hyperbolic point, then the hyperbolic lines are concurrent. Otherwise, if their common point is on the absolute conic, they are called asymptotically parallel lines, and if their common point is an outer point of the absolute conic, they are called ultraparallel lines.

Concerning the preliminaries on projective geometry we refer to [2] or [4]. The following theorem of Chasles plays an important role in our considerations, so we formulate it explicitly:

Theorem 1 (Chasles). Suppose that a triangle is different from the triangle formed by the polars of its vertices with respect to a given conic (i.e. its polar triangle). Then the triangle is perspective to its polar triangle.

Since the only involutory collineations of the real projective plane are the harmonic homologies, and a harmonic homology is an automorphism of a given conic if and only if its centre is the pole of its axis, it follows that the reflection with respect to a hyperbolic line $t$ is the restriction of the harmonic homology 


$$
\text { "tmcs-szilasi" — 2012/3/1 - 0:14 - page } 177 \text { — \#3 }
$$

whose axis is the projective line corresponding $t$, and whose centre is the pole of $t$ with respect to the absolute conic.

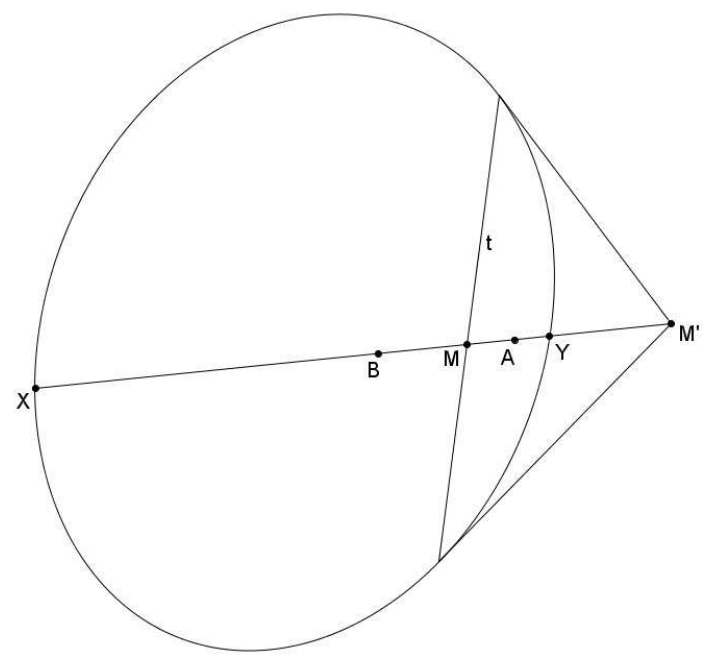

Figure 1. Reflection with respect to $t$ in the Cayley-Klein plane

Since a line is perpendicular to $t$ if and only if it is a non-pointwise fixed invariant line of the reflection with respect to $t$, the lines perpendicular to $t$ are just the lines whose corresponding projective lines pass through the pole of $t$. This means that two hyperbolic lines are perpendicular if and only if their corresponding projective lines are conjugate with respect to the absolute conic.

A point $M$ is the midpoint of the segment $\overline{A B}$ if and only if there is a reflection whose axis passes through $M$, and that sends $A$ to $B$. Let the centre of the harmonic homology corresponding to this reflection be $M_{1}$. Then $M$ and $M_{1}$ are conjugate with respect to the absolute conic and they are harmonic conjugate with respect to $A$ and $B$. This means that the quadruples $\left(A B M M_{1}\right)$ and $\left(X Y M M_{1}\right)$, where $X$ and $Y$ are the intersections of the absolute conic and the projective line corresponding to $\overleftrightarrow{A B}$ (i.e. the ends of $\overleftrightarrow{A B}$ ), are harmonic

Using some elementary facts of projective geometry, the following observations can easily be proved. For every quadruple of distinct collinear points $A, B$, $X, Y$, where $(A B)$ does not seperate $(X Y)$, there is exactly one pair of points $\left(M, M_{1}\right)$ such that both $\left(A B M M_{1}\right)$ and $\left(X Y M M_{1}\right)$ are harmonic. If $A$ and $B$ are hyperbolic points, $X$ and $Y$ are the ends of $\overleftrightarrow{A B}$, then only one member of the pair is inside the absolute conic. This point is the midpoint of the segment 


$$
\text { "tmcs-szilasi" — 2012/3/1 - 0:14 — page } 178 \text { — \#4 }
$$

$\overline{A B}$. The other point of the pair is called the outer point corresponding to the midpoint.

\section{Triangle geometry in the Cayley-Klein plane}

In the following we are going to prove some famous theorems concerning the geometry of hyperbolic triangles. Since every hyperbolic plane is isomorphic to the Cayley-Klein plane, we may restrict ourselves to the Cayley-Klein plane.

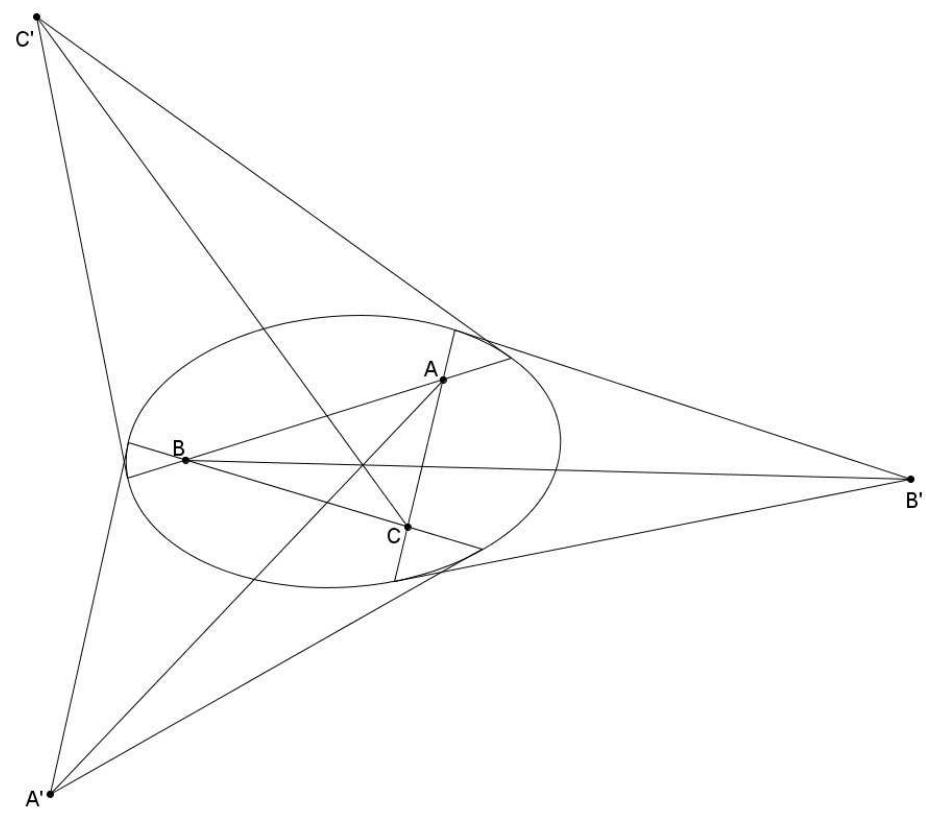

Figure 2. The altitudes of a hyperbolic triangle form a pencil.

THEOREM 2. The altitudes of every hyperbolic triangle form a pencil.

Proof. Let $A B C$ be a hyperbolic triangle. Let the poles of the projective lines corresponding to $\overleftrightarrow{B C}, \overleftrightarrow{C A}$ and $\overleftrightarrow{A B}$ be $A^{\prime}, B^{\prime}$ and $C^{\prime}$, respectively. Then the projective lines corresponding to the altitudes of $A B C$ are $\overleftrightarrow{A A^{\prime}}, \overleftrightarrow{B B^{\prime}}$ and $\overleftrightarrow{C C^{\prime}}$. By our construction the polar triangle of the projective triangle $A^{\prime} B^{\prime} C^{\prime}$ is $A B C$, 
and by the theorem of Chasles these triangles are perspective, therefore $\overleftrightarrow{A A^{\prime}}, \overleftrightarrow{B B^{\prime}}$ and $\overleftrightarrow{C C^{\prime}}$ are concurrent projective lines.

The previous proof can be found in [2]. In the next theorems we use similar techniques to deduce further important results. The key of our reasoning is the following observation.

KEY LEMma. The outer points corresponding to the midpoints of the sides of a hyperbolic triangle are collinear.

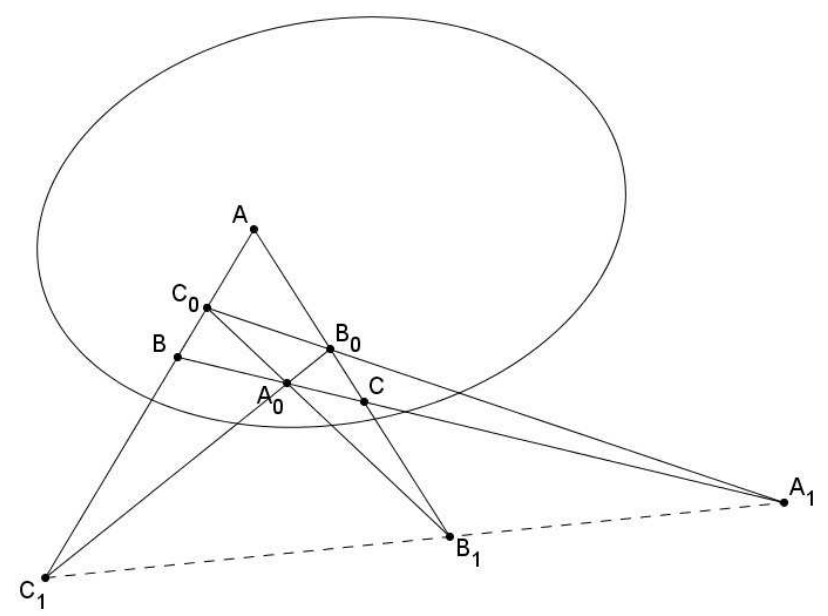

Figure 3. The outer points corresponding to the midpoints of the sides of a hyperbolic triangle are collinear.

Proof. Let the given hyperbolic triangle be $A B C$. Denote the midpoints of its sides by $A_{0}, B_{0}, C_{0}$, and the corresponding outer points by $A_{1}, B_{1}, C_{1}$.

One of the diagonal points of the complete quadrangle $C_{1} B_{1} B_{0} C_{0}$ is $A$. The other two diagonal points are incident to the line $\overleftarrow{B C}$, since $\left(C_{0} C_{1} A B\right)$ and $\left(B_{0} B_{1} A C\right)$ are both harmonic tetrads. Let these diagonal points be $\left\{A_{0}^{\prime}\right\}:=$ $\overleftrightarrow{B_{0} C_{1}} \cap \overleftrightarrow{C_{0} B_{1}}$ and $\left\{A_{1}^{\prime}\right\}:=\overleftrightarrow{B_{0} C_{0}} \cap \overleftrightarrow{B_{1} C_{1}}$. We prove that $A_{0}^{\prime}$ and $A_{1}^{\prime}$ are conjugate with respect to the absolute conic. From this our Lemma follows, since by the harmonic property of the complete quadrangles $A_{0}^{\prime}$ and $A_{1}^{\prime}$ are harmonic conjugates with respect to $B$ and $C$, so in this case $A_{0}^{\prime}=A_{0}$ and $A_{1}^{\prime}=A_{1}$. 


$$
\text { "tmcs-szilasi" — 2012/3/1 — 0:14 - page } 180 \text { — \#6 }
$$

We apply the theorem of Chasles to the triangle $A_{0}^{\prime} B_{0} C_{0}$. The polar of $C_{0}$ intersects the line $\overleftrightarrow{A_{0}^{\prime} B_{0}}$ at $C_{1}$, since $C_{1}$ and $C_{0}$ are conjugates with respect to the absolute conic. The polar of $B_{0}$ intersects the line $\overleftrightarrow{A_{0}^{\prime} C_{0}}$ at $B_{1}$, since $B_{1}$ and $B_{0}$ are also conjugates with respect to the absolute conic. This means that the intersection of $\overleftrightarrow{B_{0} C_{0}}$ and the polar of $A_{0}^{\prime}$ must be on the line $\overleftrightarrow{B_{1} C_{1}}$, so $A_{1}^{\prime}$ is on the polar of $A_{0}^{\prime}$. So it follows that $A_{0}^{\prime}$ and $A_{1}^{\prime}$ are conjugates with respect to the absolute conic, as was to be shown.

THEOREM 3. The perpendicular bisectors of the sides of a hyperbolic triangle form a pencil.

Proof. We use the notations of the previous Lemma. The perpendicular bisectors of the sides of the triangle $A B C$ are the polars of $A_{1}, B_{1}$ and $C_{1}$. Since $A_{1}, B_{1}$ and $C_{1}$ are collinear, their polars are concurrent. This proves our assertion.

THEOREM 4. The midlines of every hyperbolic triangle form a pencil.

Proof. Again, we use the notations of the Key Lemma. Since the triangles $A B C$ and $A_{0} B_{0} C_{0}$ are axially perspective (with respect to the line passing through $A_{1}, B_{1}$ and $C_{1}$ ), by the theorem of Desargues they are also centrally perspective. This means that the projective lines corresponding to the midlines of any hyperbolic triangle are concurrent.

As it is well-known, the previous theorems are true in Euclidean geometry as well. In the Euclidean case the common points of altitudes, perpendicular bisectors and midlines always exist; they are called orthocentre, circumcentre and centroid, respectively. It is also true in the Euclidean geometry that these points are collinear (if the points do not coincide), their common line is called the Euler line of the triangle. Now we find, however, an essential difference between the Euclidean and the hyperbolic case. In [3] J. R. Klus studied some concrete examples and conjectured that in hyperbolic geometry the only triangles with collinear orthocentre, circumcentre and centroid are the isosceles triangles. Applying our methods, we prove this assertion, thus we give an essentially simpler new proof of a theorem of R. Baldus [1].

THEOREM 5. The orthocentre, circumcentre and centroid of a hyperbolic triangle are collinear if and only if the triangle is isosceles. 
Proof. We keep our previous notations: the given triangle is $A B C$, the midpoints of the corresponding sides are $A_{0}, B_{0}$ and $C_{0}$, and the poles of the projective lines corresponding to the sides are $A^{\prime}, B^{\prime}$ and $C^{\prime}$. Then the orthocentre of the triangle is $\overleftrightarrow{A A^{\prime}} \cap \overleftrightarrow{B B^{\prime}}$, the circumcentre is $\overleftrightarrow{A_{0} A^{\prime}} \cap \overleftrightarrow{B_{0} B^{\prime}}$, and the centroid is $\overleftrightarrow{A A_{0}} \cap \overleftrightarrow{B B_{0}}$. Suppose that the triangle $A B C$ is not isosceles. Then $A, A^{\prime}, A_{0}$ and $B, B^{\prime}, B_{0}$ are not collinear triples of points. In this case, the orthocentre, the circumcentre and the centroid are collinear if and only if the triangles $A A^{\prime} A_{0}$ and $B B^{\prime} B_{0}$ are axially perspective. By the theorem of Desargues, it holds if and only if they are centrally perspective, i.e., if $\overleftrightarrow{A B}, \overleftrightarrow{A^{\prime} B^{\prime}}$ and $\overleftrightarrow{A_{0} B_{0}}$ are concurrent. Here, by our Key Lemma, $\overleftrightarrow{A B} \cap \overleftrightarrow{A_{0} B_{0}}$ is $C_{1}$, the outer point corresponding to the midpoint $C_{0} . C_{0}$ is incident with $\overleftrightarrow{A^{\prime} B^{\prime}}$ if and only if the polar of $C_{0}$ is incident with the intersection of the polars of $A^{\prime}$ and $B^{\prime}$. The latter point is $C$, while the polar of $C_{0}$ is the perpendicular bisector of $\overline{A B}$. If $C$ is on this line, then the perpendicular bisector, the altitude and the midline corresponding to $C$ coincide. This contradicts our assumption that the triangle is not isosceles.

Conversely, if $A B C$ is isosceles, and - for example - $\overline{B C}$ is the base of this triangle, then $\overleftrightarrow{A A_{0}}$ is the perpendicular bisector, altitude and midline of this triangle at the same time. Thus the orthocentre, the circumcentre and the centroid are incident with this line as well, so in this case the three points are collinear.

\section{References}

[1] R. Baldus, Über Eulers Dreieckssatz in der Absoluten Geometrie, Sitzungsberichte der Heidelberger Akademie der Wissenschaften, Math.-naturw. Kl. 11 (1929).

[2] H. S. M. Coxeter, Non-Euclidean Geometry, Sixth edition, Mathematical Association of America, 1998.

[3] J. R. Klus, The Euler Line in Hyperbolic Geometry, SUNY Geneseo Journal of Science and Mathematics 1, no. 1 (2000), $11-15$.

[4] J. Richter-Gebert, Perspectives on Projective Geometry, Springer-Verlag, Berlin, 2011.

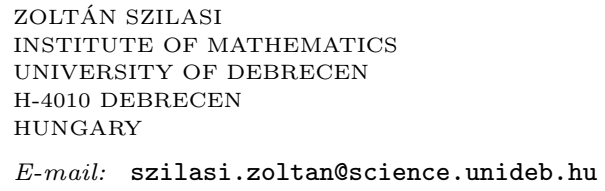

(Received June, 2011) 